\title{
CERTAIN PROPERTIES OF AN OPERATOR INVOLVING SUBORDINATION
}

\begin{abstract}
The concept of subordination can be traced back to Lindelöf since 1909, but other mathematicians like Littlewood (1925) and Rogosinski (1939) introduced the term and developed the basic theories. Subordination now plays an important role in complex analysis. The idea of univalent subordination can be stated as follows: Let $f$ and $g$ be analytic in $E$. Then $f$ is said to be subordinate to $g$, if $g$ is univalent in $E, f(0)=$ $=g(0)$ and $f(E) \subset g(E)$. We denote the subordination by $f \prec$ $\prec g$. Here, we apply a lemma of Miller and Mocanu to obtain a series of best possible subordination theorems. We also make use of an operator studied by Cho and Srivastava, and by Cho and Kim in this particular work. Thus, in this research work, we consider properties of an operator aforementioned involving subordinations with new results briefly highlighted.
\end{abstract}

Key words: analytic function, surbodination, Hadamard product, hypergeometric functions

2010 Mathematical Subject Classification: $30 C 45$

1. Introduction and Definitions. Let $\mathcal{A}$ denote the class of analytic functions of the form

$$
f(z)=z+\sum_{k=2}^{\infty} a_{k} z^{k}
$$

which are analytic in the open unit disk $E=\{z:|z|<1\}$ and normalized by $f(0)=f^{\prime}(0)-1=0$. Let $\mathrm{S}$ be the subclass of $\mathcal{A}$ consisting of analytic univalent function of the form (1).

Let $f(z)$ and $g(z)$ be analytic functions in $\mathrm{E}, f(z)$ is said to be subordinate to $g(z)$ in $E$ written $f \prec g$ or $f(z) \prec g(z)(z \in E)$, if there exist 
a Schwarz function $w(z)$, analytic in $E$ with $w(0)=0$ and $|w(z)|<1$ such that $f(z)=g(w(z)), z \in E$. It is well know that if the function $g$ is univalent in $E$, then the above subordination is equivalent to $f(0)=g(0)$ and $f(E) \subset g(E)$.

Suppose $\Psi: \mathbb{C}^{3} \times E \rightarrow E$ and let $h$ be univalent in $E$ and satisfies the second-order differential subordinations

$$
\Psi\left(p(z), z p^{\prime}(z), z^{2} p^{\prime \prime}(z): z\right) \prec h(z), z \in E .
$$

Then $\mathrm{p}(\mathrm{z})$ is called the solution of the differential subordination.The univalent function $q$ is called dominant if $p \prec q$ for all $p$ satisfying (2). A dominant $\breve{q}$ that satisfies $\breve{q} \prec q$ for all dominant $q$ of $(2)$ is said to be the best dominant of (2). The best dominant is unique up to rotation of $E$.

\section{Preliminary Lemmas.}

Lemma 1. 1] Let $g$ be a convex function in $E$ and let

$$
h(z)=g(z)+n \alpha g^{\prime}(z), z \in E,
$$

where $\alpha>0$ and $n$ is a positive integer. If

$$
p(z)=g(0)+p_{n} z^{n}+p_{n+1} z^{n+1}+\ldots, \quad z \in E,
$$

is holomorphic in $E$ and

$$
p(z)+\alpha z p^{\prime}(z) \prec h(z), z \in E,
$$

then $p(z) \prec q(z)$ and the result is sharp.

Lemma 2. 2] Let $h$ be a convex function in $E$ with $h(0)=a, 0 \neq \gamma \in \mathbb{C}$ and $\operatorname{Re} \gamma \geq 0$ if $p(z) \in H(a, n)$ and

$$
p(z)+\frac{1}{\gamma} z p^{\prime}(z) \prec h(z),
$$

then

$$
p(z) \prec q(z) \prec h(z),
$$

where

$$
q(z)=\frac{\gamma}{\gamma z^{\frac{\gamma}{n}}} \int_{0}^{z} h(t) t^{\frac{\gamma}{n}-1} d t .
$$


The function $q$ is convex and is the best dominant.

We will make use of the operator $T_{\lambda}^{n} f(z): \mathcal{A} \rightarrow \mathcal{A}$ defined as follows: if $f(z) \in \mathcal{A}$ is of the form (1), then

$$
T_{\lambda}^{n} f(z)=z+\sum_{k=2}^{\infty}\left(\frac{k+\lambda}{1+\lambda}\right)^{n} a_{k} z^{k} .
$$

The operator $T_{\lambda}^{n} f(z)=z+\sum_{k=2}^{\infty}\left(\frac{k+\lambda}{1+\lambda}\right)^{n} a_{k} z^{k}$ recently was studied by N. Cho and H. M. Srivastava [3] and N. E. Cho and T. H. Kim [4] which is closely related to the multiplier transformation studied by Flet [5]. For detail analysis of convolutions of operators related to multiplier transformations see [6].

It is easily verify that $T_{\lambda}^{n} f(z)$ satisfy a three term recurrence relation given by

$$
T_{\lambda}^{n+1} f(z)=(1-\lambda) T_{\lambda}^{n} f(z)+z \lambda\left[T_{\lambda}^{n} f(z)\right]^{\prime} .
$$

Motivated by the work of D. Bansal and R. K. Raina [7] we obtain certain subordination properties involving the linear operator (3). Examples and corollaries are stated as consequences of the main results and some of them related to other well known results.

\section{Main Results.}

Theorem 1. Suppose $f$ is of the form (1) and satisfies the differential subordination

$$
\left[T_{\lambda}^{n+1} f(z)\right]^{\prime} \prec h(z), \quad z \in E, \lambda>0, n \in N \cup\{0\} .
$$

Then

$$
\left[T_{\lambda}^{n} f(z)\right]^{\prime} \prec q(z)
$$

where $h(z) \in \mathcal{P}$ and is given by

$$
h(z)=\left(\frac{1+A z}{1+B z}\right)^{r},(|A| \leq 1 ;|B| \leq 1 ; A \neq B ; 0<r \leq 1),
$$

and $q(z)$ is the best dominant given by

$$
\left\{\begin{array}{c}
\left(\frac{A}{B}\right)^{r} \sum_{j \geq 0}^{\infty} \frac{(-r) j}{j !}\left(\frac{A-B}{A}\right)^{j}(1+B z)^{-1}{ }_{2} F_{1}\left(j, 1 ; 1+\frac{1}{\lambda} ; \frac{B}{1+B z}\right), B \neq 0 \\
{ }_{2} F_{1}\left(-r, \frac{1}{\lambda} ; 1+\frac{1}{\lambda} ;-A z\right), \quad \text { if } B=0 .
\end{array}\right.
$$


The function $q(z)$ is convex and is the best dominant. While

$$
{ }_{2} F_{1}(a, b, c: z)=1+\frac{a b}{c} \frac{z}{1 !}+\frac{a(a+1) b(b+1)}{c(c+1)} \frac{z^{2}}{2 !}+\cdots
$$

denote the Guassian hypergeometric function.

Proof. Differentiating (4) we have the following relations

$$
\left[T_{\lambda}^{n+1} f(z)\right]^{\prime}=\left[\left[T_{\lambda}^{n} f(z)\right]\right]^{\prime}+\lambda z\left[\left[T_{\lambda}^{n} f(z)\right]\right]^{\prime \prime} \prec h(z) .
$$

Relating (8) with (5), we have

$$
\left[T_{\lambda}^{n} f(z)\right]^{\prime}+\lambda z\left[T_{\lambda}^{n} f(z)\right]^{\prime \prime} \prec h(z) .
$$

Let

$$
p(z)=\left[T_{\lambda}^{n} f(z)\right]^{\prime} .
$$

In view of (9) and (10) we have the following differential subordination

$$
p(z)+\lambda z p^{\prime}(z) \prec h(z)=\left(\frac{1+A z}{1+B z}\right)^{r}, z \in E .
$$

Applying Lemma 2 we have

$$
\begin{gathered}
p(z) \prec q(z)=\frac{1}{\lambda z^{\frac{1}{\lambda}}} \int_{0}^{z} h(t) t^{\frac{1}{\lambda}-1} d t \\
=\frac{1}{\lambda z^{\frac{1}{\lambda}}} \int\left(\frac{1+A t}{1+B t}\right)^{r} t^{\frac{1}{\lambda}-1} d t .
\end{gathered}
$$

Evaluating the integral in the manner of 8 , with slight modifications, we have the following

$$
t^{\frac{1}{\lambda}-1}\left(\frac{1+A t}{1+B t}\right)^{r}=\left(\frac{A}{B}\right)^{r} t^{\frac{1}{\lambda}-1}\left(1-\frac{A-B}{A(1+B t)}\right)^{r} .
$$

Expanding the above binomial expansion, see 8 and using integral and transformation formulas [9]:

$$
\int_{0}^{1} t^{b-1}(1-t)^{c-b-1}(1-z t)^{-a} d t=\frac{\Gamma(b) \Gamma(c-b)}{\Gamma(c)}{ }_{2} F_{1}\left(a, b ; c ; \frac{z}{z-1}\right)
$$




$$
{ }_{2} F_{1}(a, b ; c ; z)=(1-z)^{-a}{ }_{2} F_{1}\left(a, c-b ; c ; \frac{z}{1-z}\right)
$$

where ${ }_{2} F_{1}(a, b ; c ; z)$ is the Gaussian hypergeometric function, thus we obtain

$$
q(z)=\left(\frac{A}{B}\right)^{r} \sum_{j \geq 0}^{\infty} \frac{(-r) j}{j !}\left(\frac{A-B}{A}\right)^{j}(1+B z)^{-1}{ }_{2} F_{1}\left(j, 1,1+\frac{1}{\lambda} ; \frac{B}{1+B z}\right),
$$

if $B \neq 0$.

For $B=0$,we have

$$
q(z)=\frac{1}{\lambda z^{\frac{1}{\lambda}}} \int_{0}^{z}(1+A t)^{r} t^{\frac{1}{\lambda}-1} d t
$$

and following the above processes we have; $q(z)={ }_{2} F_{1}\left(-r, \frac{1}{\lambda} ; 1+\frac{1}{\lambda} ;-A z\right)$. Using Lemma 2 for $\gamma=\frac{1}{\lambda}, n=1$ we have $\left[T_{\lambda}^{n} f(z)\right]^{\prime} \prec q(z) \prec h(z)$, where

$$
q(z)=\frac{1}{\lambda z^{\frac{1}{\lambda}}} \int_{0}^{z} h(t) t^{\frac{1}{\lambda}-1} d t=\frac{1}{\lambda z^{\frac{1}{\lambda}}} \int_{0}^{z}\left(\frac{1+A t}{1+B t}\right) t^{\frac{1}{\lambda}-1} d t .
$$

Evaluating the above integral we have our desired result.

Example 1. Suppose $r=1, B=0$ and $A=1-2 \beta$, then from Theorem 1 we have that if

$$
\left[(1-\lambda) T_{\lambda}^{n} f(z)+z \lambda\left[T_{\lambda}^{n} f(z)\right]^{\prime}\right]^{\prime} \prec 1+(1-2 \beta) z
$$

then it implies that

$$
\left[T_{\lambda}^{n} f(z)\right]^{\prime} \prec 1+\frac{(1-2 \beta) z}{\lambda+1} .
$$

Theorem 2. Let $q(z)$ be a convex function in $E$ with $q(0)=1$ and let $h(z)=q(z)+\lambda z q^{\prime}(z),(z \in E)$. Suppose $f \in \mathcal{A}$ and satisfies the differential subordination

$$
\left[T_{\lambda}^{n+1} f(z)\right]^{\prime} \prec h(z),
$$

then

$$
\left[T_{\lambda}^{n} f(z)\right]^{\prime} \prec q(z),(z \in E) .
$$

This result is sharp. 
Proof. Consider again $p(z)=\left[T_{\lambda}^{n+1} f(z)\right]^{\prime}$ and making use of $(8), 10$ and by our hypothesis, we have

$$
p(z)+\lambda z p^{\prime}(z) \prec h(z)=q(z)+\lambda z q^{\prime}(z) .
$$

Applying Lemma 1 we have

$$
p(z) \prec q(z),
$$

and by implication we have

$$
\left[T_{\lambda}^{n+1} f(z)\right]^{\prime} \prec q(z),
$$

this gives our desired result.

Suppose we set $q(z)=\frac{1+(1-2 \beta) z}{1-z}, \beta>0$ in Theorem 2 , then we have the following:

Example 2. If

$$
\left[(1-\lambda) T_{\lambda}^{n} f(z)+z \lambda\left[T_{\lambda}^{n} f(z)\right]^{\prime}\right]^{\prime} \prec \frac{1+2(\lambda-\beta-\lambda \beta) z-(1-2 \beta) z^{2}}{(1-z)^{2}},
$$

$f \in \mathcal{A}$, then it implies that

$$
\left[T_{\lambda}^{n} f(z)\right]^{\prime} \prec \frac{1+(1-2 \beta) z}{1-z}, z \in E .
$$

Theorem 3. Let $t$ be a convex function in $E$, with $t(0)=1$, suppose $\lambda>0, n \in N \cup\{0\}$ and let

$$
h(z)=t(z)+z t^{\prime}(z),(z \in E) .
$$

If $f \in \mathcal{A}$ satisfies the differential subordination

$$
\left[T_{\lambda}^{n} f(z)\right]^{\prime} \prec h(z)
$$

then

$$
\frac{\left[T_{\lambda}^{n} f(z)\right]}{z} \prec t(z)
$$

and this result is sharp.

Proof. Let

$$
\frac{\left[T_{\lambda}^{n} f(z)\right]}{z}=\mu(z) .
$$


Differentiating (13) with respect to $z$, we obtain

$$
\left[T_{\lambda}^{n} f(z)\right]^{\prime}=\mu(z)+z \mu^{\prime}(z)
$$

relating (14) with 12 , then the differential subordination 12 implies

$$
p(z)+\lambda z p^{\prime}(z) \prec \mu(z)+\lambda z \mu^{\prime}(z),
$$

and applying Lemma 1 we obtain

$$
p(z) \prec t(z)
$$

which implies the result

$$
\left[T_{\lambda}^{n} f(z)\right]^{\prime} \prec t(z)
$$

and the result is sharp.

Example 3. Suppose we have $n=0, t(z)=\frac{1+(1-2 \beta) z}{1-z}, 0 \leq \beta<1$, then we have the following:

$$
[f(z)]^{\prime} \prec \frac{1+2(\lambda-\beta-\lambda \beta) z+(1-2 \beta) z^{2}}{(1-z)^{2}}, \quad f \in \mathcal{A},
$$

then it implies that the following subordination holds true

$$
\frac{f(z)}{z} \prec \frac{1+(1-2 \beta) z}{1-z} .
$$

Acknowledgment. The first author was visiting as a research fellow with the School of Mathematical Sciences, Universiti Kebangsaan Malaysia. (TETfund, Nigeria, is acknowledged for the partial sponsorship). The second author is supported by AP-2013-009. The authors would like to thank the referee for the informative comments to improve the manuscript.

\section{References}

[1] Miller S. S., Mocanu P. T. Differential Subordinations: Theory and Applications. Marcel Dekker, New York, NY, USA, 2000.

[2] Hallenbeck D. J., Ruscheweyh S. B. Subordination by convex functions. Proc. Amer. Math. Soc., 1975, vol. 52, pp. 191-195. 
[3] Cho N. E., Srivastava H. M. Argument estimates of certain analytic functions defined by a class of multiplier transformations. Math. Comput. Modelling, 2003, vol. 37, no. 1, pp. 39-49.

[4] Cho N. E., Kim T. H. Multiplier transformations and strongly close-toconvex functions. Bull. Korean Math. Soc., 2003, vol. 40, no. 3, pp. 399410.

[5] Flett T. M. The dual of an inequality of Hardy and Littlewood and some related inequalities. J. Math. Anal. Appl., 1972, vol. 38, pp. 746-765.

[6] Li J. L., Srivastava H. M. Some inclusion properties of the class $P_{\alpha}(\beta)$. Integral Transform and Spec. Funct., 1999, vol. 8, pp. 57-64.

[7] Bansal D., Raina R. K. Some subordination theorems associated witha new operator. Le Mathematiche, 2010, vol. 25, pp. 33-34.

[8] Prajapat J. K., Raina, R. K. Subordination results for certain classes of multivalently analytic functions with a convulotion structure. East Asian J. Math., 2009, vol. 25, pp. 267-276.

[9] Abramowitz M., Stegan I. A. Handbook of Mathematical Functions with Formulas, Graphs and Mathematical Tables. Dover Publications, New York, 1971.

Received February 15, 2015.

In revised form, November 15, 2015.

Plateau State University

Bokkos, Plateau State, Nigeria;

Universiti Kebangsaan Malaysia

43600 UKM Bangi Selangor DE, Malaysia

E-mail: philipajai2k2@yahoo.com

Universiti Kebangsaan Malaysia

43600 UKM Bangi Selangor DE, Malaysia

E-mail: maslina@ukm.edu.my 